\title{
Architecture of Automation Control Model for Robotics
}

\author{
Nisha P. Zode, \\ Kamla Nehru Mahavidyalaya, \\ Sakkardara, Nagpur (MS), India
}

\author{
Bhaskar Y. Kathane, \\ VMV, JMT, JJP Science College, \\ Wardhaman Nagar, Nagpur (MS), India
}

\begin{abstract}
A robot is a device which is behaved like an artificial agent and it is also called as an electro mechanical machine which based on autonomous control. As the robotic based on various control paths which controls the robots by planning, Electronic sensor, Digital signals, Computer mechanics, Coordination, Actuation, Learning and adaptation. In this paper presents the new robot control architecture for Intelligent Robot navigation to navigate its different direction of the wall avoiding obstacle. In contrast to standard formulation of robot behaviors, in proposed architecture presented which shows the modeling of robot behaviors with using the Autonomous robot Control.
\end{abstract}

\section{Keyword}

Robot, Control, Behavior based, autonomous.

\section{INTRODUCTION}

The steady Increase of air traffic flow over the next 20years is expected due to the economic growth and demographic changes in the emerging economic nations. Since Aviation safety has been highly guaranteed throughout the process of design, manufacturing and operation of an aircraft, the fatal accident rate has been kept low for the past 20 years. However it is concerned that increasing flight will lead to more accident unless steps are taken to drastically reduce accident rates [1]. As for the guidance and control technologies, flight automation such as autopilot, autothrottle and Flight Management System (FMS) has greatly contributed to increase the flight safety. However, these functions cannot adapt themselves to maintain their performance in the case of unexpected failure in such Case pilot have to manage the difficult situation which may increase their workload and may cause accidents. It is expected that a fault tolerant control or a resilient control can extend the limit of automatic control system [2]. In recent years, concept of automatic control has achieved a very important position advancement of modern science. Automatic control systems have played a very important role in the advancement and improvement of engineering skills [3]. The most recent example of automatic control is robotics.

Robotics is an extremely dynamic field with thriving advancement in technology Along with other emerging technologies such as information technology, biotechnology, nanotechnology robotics will contribute to increase opportunities for economic growth and greatly affect future generation with substantial social and economic impact[4].An autonomous robot is a robot that performs behaviours or tasks with a high degree of autonomy, which is particularly desirable in fields such as space exploration, cleaning floors, mowing lawns, and waste water treatment[5].Autonomous robots are intelligent machines capable of performing tasks in the world by themselves, without explicit human control.
Robots are composed of movable physical structures, a power system (hydraulic, electrical or pneumatic), sensor system, a motor and a "brain" that tells its parts what to do. They may have an "arm," called an end effect or that can use tools or be grippers. They will usually have a sensor which enables them to receive information about what is happening around them. This Thesis is contribute with the feature of robotic that is based on the control system. As how we can contribute the develop architecture and hierarchical representation of the command and manage a swarm mobile robot. This Model Contain topological and geometrical representation with the help of control system based on various control path planning, Electronic sensor, Digital components mechanics, Coordination, Actuation, Learning and adaptation. The topology of the space to be inspected is known in advance and the robot is able to navigate along different direction of the wall while avoiding the different obstacle due to structure of building.

\section{RESEARCH OBJECTIVES}

The important objective of the research is to provide a computer base solution to control robotics based on different sensors, software programs and hardware. That is inspected the robotic control in advance with the help of actuator and sensor which help the mobile robot to navigate its different direction of the wall while avoiding different obstacle due to structure of building. This is achieved through developing a Robotics control model.

\section{ROBOTICS CONTROL MODEL}

Making systems of various kinds perform well is what automatic control is all about. The interplay between robotics and control theory has a rich history extending back over half a century. We begin this section of the report by briefly reviewing the history of this interplay, focusing on fundamentals, how control theory has enabled solutions to fundamental problems in robotics and how problems in robotics have motivated the development of new control theory [6]. Robotic control architecture the robot first plans a solution for the task by reasoning about the outcome of its actions and then executes it. Control process goes through a sequence of sensing, model update, and planning steps. Path planning, sometimes called "Motion Planning", is the act of finding a path to go from location A to B. A global path planner usually generates a low-resolution high-level path from $\mathrm{A}$ to $\mathrm{B}$, avoiding large obstacles and dealing with navigation around the area. Local path planning usually gives a high-resolution low-level path only over a segment from global path $\mathrm{A}$ to $\mathrm{B}$, avoiding small obstacles and dealing with motion planning: angles of turn, appropriate velocities, etc. The planning module is also in charge of sending the goals of the problem that is being solved to the monitoring in order to supervise the execution of the proposed solution. In case of replanting, the planning module (that already knows the domain) will receive the high level state from the execution 
module. Then the entire process will begin again using this state as the new initial state, electronic sensor device which provides information about the physical world. Digital Computation which gives computationally any type of calculation that is the robotic path detected analog or digital method. Mechanics is the part which gives the robot to move forward or backward in the location. Here we describe the nature of the robot how the robot co-ordinate with the obstacle with the help of actuator .Co-ordinate Level receives command to perform predetermined specific control task from the control manager to the above. It provides the appropriate sequence control and identification .The actuator which used in robotic to move the muscles of the robots with the help of Beep, Motors or LED Lights which given in below fig 1.

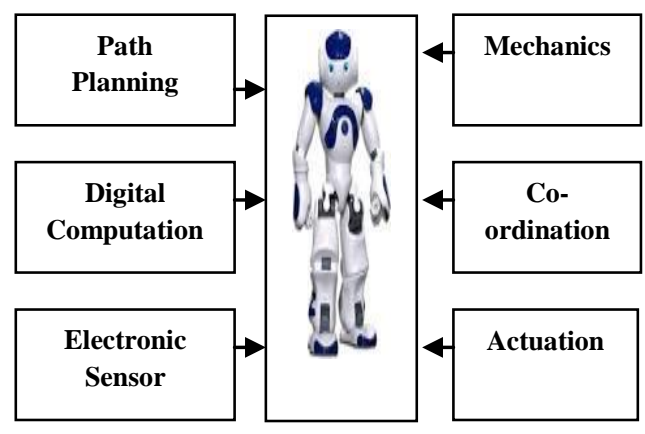

Fig 1: Robotic Model

The Robotic Model which gives the study us for Robot Most of the papers on the control of rigid robots start with the wellknown Lagrangian model and attempt to apply standard techniques to this model, be they in the nature of adaptive control, feedback linearization etc. This area is by now saturated, and there is not much left to be done in contrast. The problem of designing robot in such a way that they are easy to control has received relatively little attention one of the recent trends in robotics is the use of direct drive motors. "Direct drive" refers to the fact that the links of the robot are coupled directly to the respective motor shafts without going through any gearing. The advantage of such a scheme is that one immediately eliminates the effects of backlash, joint flexibility, etc. Which arise from the use of gears, and can therefore aspire to more accurate and repeatable performance from the robot.

\subsection{Autonomous Control Model}

An autonomous system is a system that can perform some desired task within an unstructured environment without human intervention [7]. Our research on autonomous systems is focused on making use of measurements from various sensors in order to understand what happens and gain situational awareness. The applied research is very important when it comes to autonomous systems and to mention a few examples that we have been working on, we have unmanned area vehicles, active safety systems within the automotive industry and fighter aircraft navigation. The control of autonomous robots involves a number of subtasks. Autonomous control Model control the robotic architecture based on the Understanding and modeling mechanism of robot. Through the specific subtasks that is using Kinematics, Dynamics and odometer. The Kinematics which based on the Forward kinematics describes how the robots joint angle configurations translate to locations in the world and Inverse kinematics computes the joint angle configuration necessary to reach a particular point in space. Secondly the Control based on reliable Control of Actuator i.e. to get a particular robot actuator to a particular location it is important to apply the correct amount of force or torque to it. In the Third step we goes to the task of Specific motion Path planning addresses the task of computing a trajectory for the robot such that it reaches the desired goal without colliding with obstacles. In the Fourth Control we used the sensor which used to perceive the environment. In the Fifth Step we Copying the noise and uncertainty robot systems in intelligent environments have to deal with sensor noise and uncertainty i.e. the sensor uncertainty, Non-observability and Action Uncertainty and the Sixth step which gives us a flexible control by using all these steps without any obstacle and manage the mobile robot which shown the control of autonomous mobile robot in the below fig 2 .

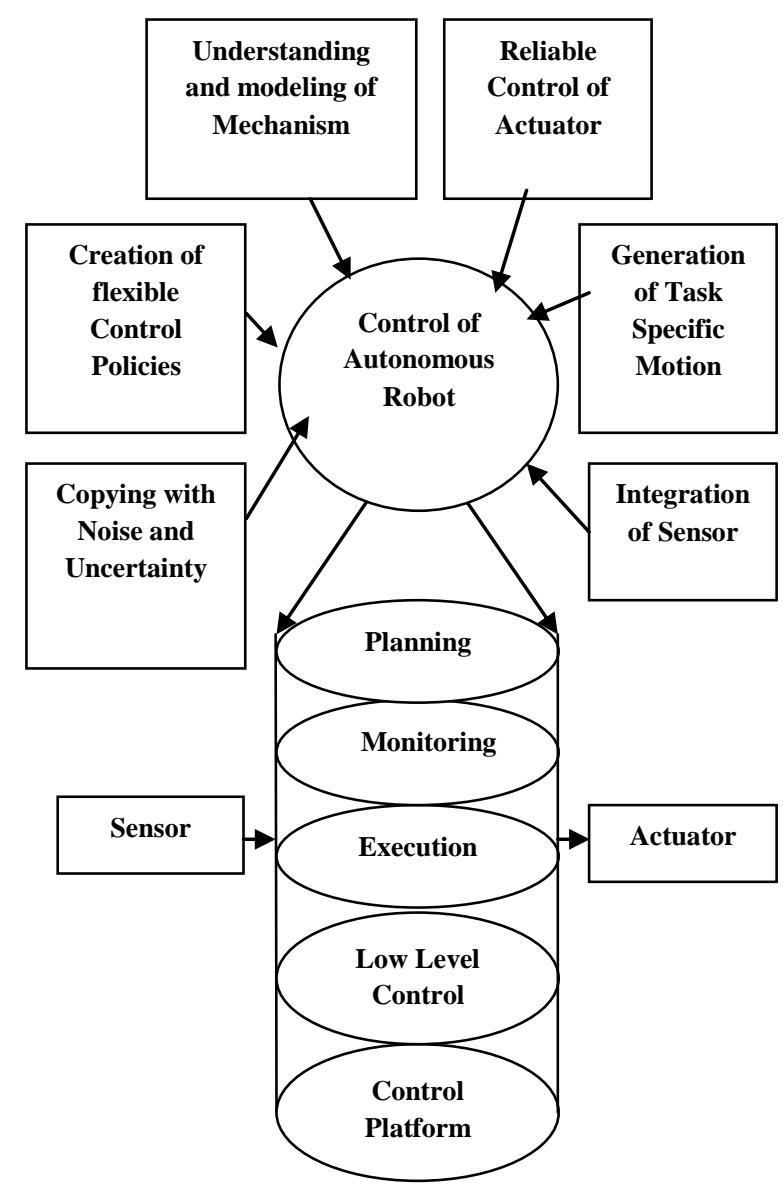

Fig 2: Autonomous Control Model

\section{- Planning}

Automation of planning processes such as these has been a central problem in the field of artificial intelligence for more than 30 years, and a number of important approaches, including state space search and hierarchical task decomposition, have be characterized as a plan executor. This loop uses filtered perception data to assess the progress of the system through a preplanned sequence of states. Planning is defined as Reasoning about how to achieve a goal. It employs a suitable model map connecting sequence of states from an initial state to a goal state. 


\section{- $\quad$ Monitoring}

It is difficult to predict the result of executing an action in non-deterministic planning domains such as the robot control ones, where the environment is dynamic. So, we need a module that supervises the execution and detects when it can not continue with the execution of the initial plan. Monitoring can be done at the low level or at the high level. For our work, we focus on high level supervision using a monitoring module. This module carries out the supervision of the plan execution and the achievement of goals by receiving information from the execution module.

\section{- $\quad$ Execution}

While architectures have an essential role in the designed specification of a robotic control application, they also play a significant role in the run-time execution of robotic control software. For robotic control systems, run-time execution typically includes issues such as realtime response, appropriate goal-directed behavior, and reliable reactivity to environmental changes. Once a plan is set up, it should be faithfully executed. Execution with Verification map from the input command and it expected normal response to success/failure. As long as the execution is successful, it continues the goal is achieved.

\section{- Low Level Control}

To achieve robotic-device independence, the actuators/ sensors management is all done by the low level control layer that implements the basic control skills. This module receives low level action requests and sends the appropriate commands to the robot actuators, handling the corresponding communication with the control platform server. In other words, this module provides a set of basic skills that compose a low-level control server interface that is going to be used by the execution module. This module is also in charge of obtaining the sensor readings from the robot after the execution of each actuator command.

\section{- Control Platform}

The main function of the module is to control the situation. The chosen control platform for commanding the robot is Player. Player is a (TCP) network server that provides an interface for robot device (sensors/actuators) control, designed to be language and platform independent. Player is a (TCP) network server that provides an interface for robot device (sensors/actuators) control, designed to be language and platform independent.

\subsection{Hierarchical Control Model}

Diverse, complex, and adaptive animal behaviors are achieved by organizing hierarchically structured controllers in motor systems. The levels of control progress from simple spinal reflexes and central pattern generators through to executive cognitive control in the frontal cortex. Various types of hierarchical control structures have been introduced and shown to be effective in past artificial agent models, but few studies have shown how such structures can self-organize. This study describes how such hierarchical control may evolve in a simple recurrent neural network model implemented in a mobile robot. Hierarchical control model which based on the node which forms of network control system? It includes a shape like tree in which each node operates independently, performing tasks from its superior node, commanding tasks of its subordinate nodes, sending abstracted sensations to its superior node, and receiving sensations from its subordinate nodes [8]. The Scribbler has two IR obstacle sensors (under the light sensors) that return a binary value of 0 or 1 . Return value 1 if IR Light is bouncing towards obstacle and return value 0 if there is no obstacle that is nothing in front of the emitter or detector and get the sensation result. This hierarchical represent us the architecture or representation of the Sensor Control in the Hierarchical way given in fig. 3

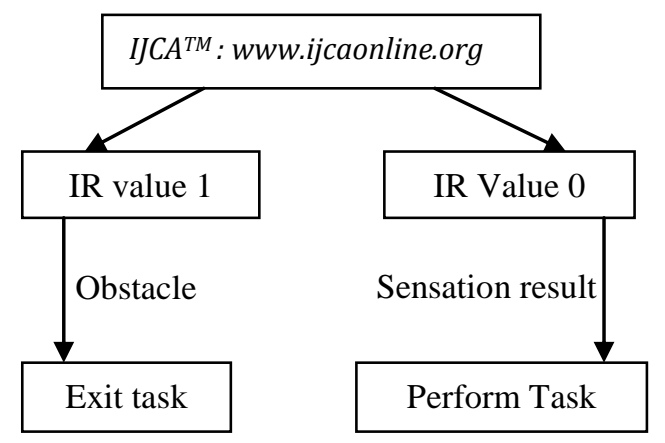

Fig 3: Hierarchical Control Model

\subsection{Behavioral Control Model}

Being unable to achieve reliable implementation of conventional approach for each and every intelligent mobile robot being developed, the robotic community turned towards new solutions. These new concepts should have provided frameworks able to tackle problems that conventional approach had not been able to. The brand new paradigm emerged in the 1980's and it was named the Behavior Based Robotics (BBR). The first proposal in the BBR style emerged in the mid 1980's. Namely, Prof. Rodney Brooks of MIT's Artificial Intelligence Laboratory proposed a new solution for mobile robot developing. In his seminar paper, Brooks argues that use of traditional hierarchical structure led researchers in the wrong direction of the main research goal, which is to build a mobile robot able to operate autonomously in the environment [9].Namely, the dominant focus on the planning system and explicit symbolic representational knowledge is timely and computationally costly, restraining robotics community to tackle those problems for the time being. $\mathrm{He}$ advocated the use of layered, but not hierarchical, architecture that could provide a mobile robot with autonomous capabilities. The low level layers are built for obstacle avoidance, while the higher layers for more abstract actions. Behavioral Model it is based on the collection of behavior Behaviors are processes or control laws that achieve and/or maintain goals. For example, 'avoid-obstacles' maintains the goal of preventing collisions; 'go-home' achieves the goal of reaching some home destination. In behavior-based control architecture the robot's actions are determined by a set of parallel, reactive behaviors which map sensory input and state to actions. Reactive, behavior-based control combines relatively simple behaviors, each of which achieves a particular subtask, to achieve the overall task of behavioral control. 


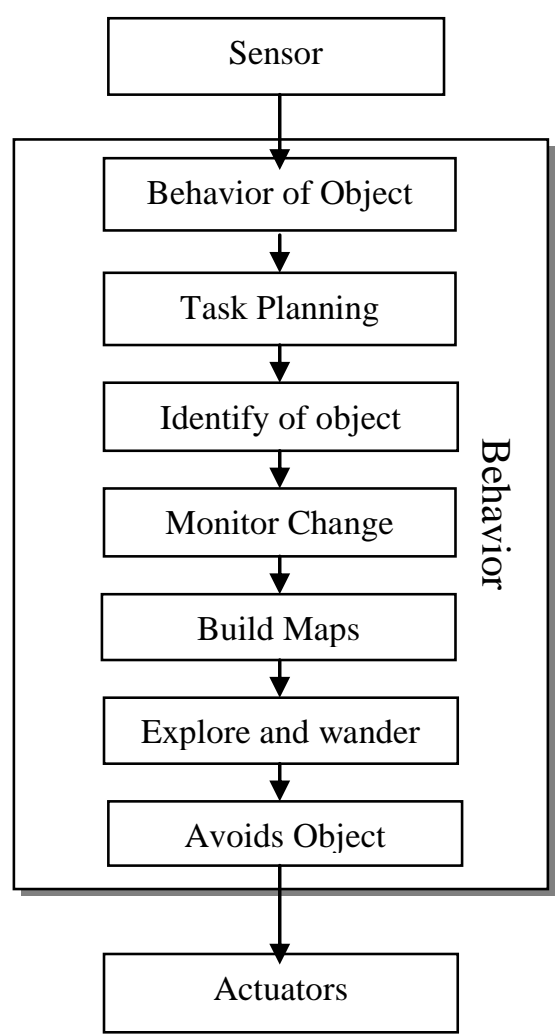

Fig 4: Behavioral Control Model

Behavior based Control which gives the behavior of the mobile robot as any object want to place the robot. Identify the behavior of object. Therefore techniques from the qualitative theory of behavioral systems can be used to design and tune autonomous robot architectures. We demonstrate these ideas in two implementations. In a first case hierarchical implementation of the same autonomous control model with the help of actuator and sensor which gives the control to avoid the obstacle. In second case, visual sensory information is integrated to achieve target acquisition and obstacle avoidance in an autonomous robot minimizing the known problem of spurious states.

\section{CONCLUSIONS}

This Paper Presented has design architecture of the robotic control and the reconfigurable autonomous control robotic system suggesting navigating mobile robotic along different direction of the wall while avoiding different the obstacle due to structure of building. Various techniques have emerged to develop the science of robotics and robots robot that is "autonomous" does not need a person to operate it more importantly, it must also have the human ability to actually "learn" from its experiences and mistakes. This is an important goal for many designers. We are currently working on the modules of autonomous control improving the control process to navigate the mobile robotics with its direction avoiding obstacle is also a part of our future work. Our medium terms goals including aspects of improving the control architecture and navigate along different direction of the wall while avoiding the different obstacle due to structure of building.

\section{ACKNOWLEDGEMENT}

We are very much thankful to Dr. P. K. Butey, Head, Associate Professor, Kamla Nehru Mahavidyalaya, Nagpur, for his valuable inputs, constant guidance and his extensive support an encouragement for this work.

\section{REFERENCES}

[1] Robotics: State of the Art and Future Challenges

[2] S. suzuki Y.Yoshimatsu K.Miyaji Dept of aeronautics and astronatics

[3] ieeexplore.ieee.org > ... > Intelligent Control, 1990. Pr by PJ Antsaklis - 1990 - Cited by 67 - Related articles

[4] Robotics: State of the Art and Future Challenges

[5] En.wikipedia.org/wiki/Autonomous robot

[6] Mark W. Spong and Masayuki Fujita Control in Robotics

[7] Autonomous Robots: From Biological Inspiration to Implementation and Control

[8] R. W. Brockett. "Asymptotic stability and feedback stabilization," in Differential Geometric Control Theory, R.W. Brockett, R.S. Millman, and H. J. Sussmann, eds. Boston-Basel-Stuttgart: Birkhauser, 1983, pp. 181191,By George A. Bekey

[9] Brooks (1986): "A robust layered control system for a Mobile robot IEEE journal. 